\title{
Clustering Nodes Selection Mechanism in Energy Heterogeneous Farmland Wireless Sensor Networks
}

\author{
Dayong Liu ${ }^{1,2, a}$,Chunjiang Zhao ${ }^{2,3, b^{*}}$, Huarui $\mathrm{Wu}^{2,3, \mathrm{c}}$ and Feifei $\mathrm{Li}^{2,3, \mathrm{~d}}$ \\ ${ }^{1}$ College of Computer Science and Technology, Beijing University of Technology, Beijing, China \\ ${ }^{2}$ Beijing Research Center for Information Technology in Agriculture, \\ Beijing Academy of Agriculture and Forestry Sciences, Beijing, China \\ ${ }^{3}$ National Engineering Research Center for Information Technology in Agriculture, Beijing, China \\ aliudyliudy@126.com, ${ }^{b^{*}}$ zhaocj@nercita.org.cn(corresponding author), ${ }^{c}$ wuhr@nercita.org.cn, \\ ${ }^{d}$ liff@nercita.org.cn
}

\begin{abstract}
Keywords: Wireless Sensor Networks, Energy Heterogeneous, Selection Mechanism, Farmland Abstract. Solar-powered nodes have been employed in farmland wireless sensor networks to prolong the lifetime, which can increase the cost at the same time. The energy acquisition mode of solar-powered node is intermittent and nonlinear, because it is easy to be influenced by the external factors. In this paper, a fuzzy prediction method of the solar energy using the weather forecast is proposed, and then a fuzzy selection method to distinguish the different types of nodes is proposed. Simulation results show that the proposed method can improve the network throughput and reduce the energy consumption of nodes.
\end{abstract}

\section{Introduction}

In the application of wireless sensor networks (WSN) for farmland monitoring, the traditional node usually uses battery power, today with the maturity of solar technology, the application of solar energy is also more and more. Solar panels will increase the cost of deploying and also extend the survival period of WSN. Therefore, energy heterogeneous nodes, including solar-powered and battery powered nodes, are often used in the deployment of farmland wireless sensor network (FWSN).

Both monitoring area and the number of nodes is large in FWSN, so hierarchical routing protocol is usually used. The key of the layered protocol is the selection of cluster nodes ${ }^{[1]}$. Solar energy nodes are often chosen as cluster nodes because of their ability to charge. However, the charging characteristics of the solar-powered nodes change with the weather, and its energy acquisition has the characteristics of intermittent and nonlinear ${ }^{[2]}$.

Based on the fuzzy theory, a new method is proposed to predict the energy of solar-powered nodes according to the weather forecast information. Finally, according to the forecast of the energy change factor and other indicators, in the network layer of the structure of the building, a clustering node selection mechanism is proposed.

\section{Related Works}

Route protocol of WSN is divided into two types: flat structure and hierarchical structure. Hierarchical route has the advantage of saving energy, so it is more suitable for the network with a large number of nodes ${ }^{[3]}$. A new network topology control strategy is proposed in this paper, which combines the remaining energy of the nodes in the network with the availability of the solar energy ${ }^{[4]}$.

Solar energy is an important factor in the selection of cluster nodes. The current energy prediction method of solar-powered supply board can be divided into two categories, which are short-term and long-term prediction ${ }^{[5]}$. The time series forecasts is used as criterion in an approach we have elaborated to detect the long-term correlation in solar irradiation series ${ }^{[6]}$. The method of predicting solar radiation is presented based on satellite data/imagery. Literature pointed out the key factors of influence of solar power panels including clouds, temperature, humidity, wind and other, a prediction 
method based on support vector machine (SVM) model of short-term generation is proposed in the paper.

The above prediction research on the solar energy is related to the solar power station, which is failed to the related application of WSN. Accurate weather forecast data and meteorological satellite cloud image/data are lacked due to the remote field environment. The above- mentioned node selection algorithm is no longer applicable to heterogeneous WSN, so a method of multiple factors is needed.

\section{Cluster Node Selection in Heterogeneous Energy Mode}

Cluster node selection factor analysis is the basis for the cluster node selection of WSN.

\section{Cluster Node Selection Factor Analysis.}

According to the difference of the power supply between the solar node and the battery node, the optimal cluster node is selected in the network. The remaining energy, the node type, the node degree and the charge coefficient are considered as the main factors to be considered in the selection of the optimal cluster nodes. Node type is a battery powered node or a solar powered node. Node degree refers to the number of nodes in the neighborhood, and the calculation method is omitted.

\section{Node Remaining Energy.}

The remaining energy of a heterogeneous node in a wireless sensor network is composed of the past remaining energy, the loss energy and the supplementary energy, which are shown in formula 1 and 2:

$$
\begin{aligned}
& Q(t+\Delta t)=Q(t)-Q_{\text {Loss }}(t)+Q_{r}(\Delta t) \\
& Q_{r}(\Delta t)= \begin{cases}0 & \text { Battery Power Node } \\
\int_{t}^{t+\Delta t} W(x) d x & \text { Solar Power Node }\end{cases}
\end{aligned}
$$

When the energy is reduced to 0 , the solar node can't work. When the weather is bad, such as the rainy season, foggy days, the energy supplement of solar-powerd node is extremely weak. So we need to find a way to more accurately predict charging capacity of solar energy node in the next few days.

\section{Charge Coefficient.}

The weather forecast information can be divided into three types: numerical, satellite cloud images and text. Due to the remote field environment, the future numerical value of clouds and satellite data/imagery are lesser. Weather forecast in the text information include: sunny, cloudy, overcast, fair to cloudy, cloudy to overcast and so on, which can reflect the situation of the cloud cover.

We put forward a kind of fuzzy method to deal with the text type weather information, which contribute to transform it into the numerical information of the charge coefficient. $A_{i}$ expresses the weather factors, $A_{i} \in A, \quad i=1, \ldots, n . A$ is represented as a set of $A_{i}$.

The fuzzy relation matrix $\mathrm{R}$ of weather forecast information is defined 3.

$$
R=A \times B, A_{i} \in A, \quad i=1, \ldots, n, B_{j} \in B, \quad j=1, \mathrm{~K}, m
$$

$B_{j}$ : Charge coefficient, $B_{j} \subset[0,1] ; B$ is output about all charge coefficient set. $B_{j}$ represents the degree of occlusion of the light. If the value of $B_{j}$ is higher, the weaker the charging ability is.

Future weather forecast information is acted as input data $A^{\prime}$, as shown in Table 1 . Because the weather is a multi-attribute relationship, we use the maximum - minimum synthesis method to calculate. Finally, according to $B^{\prime}=A^{\prime}$ o $R, B^{\prime}$ can be solved to get the future charge factor.

Table1. Weather term fuzzy assignment

\begin{tabular}{|l|l|l|l|l|l|l|l|l|l|l|l|}
\hline & 0 & 0.1 & 0.2 & 0.3 & 0.4 & 0.5 & 0.6 & 0.7 & 0.8 & 0.9 & 1 \\
\hline Sunny & 0.9 & 0.1 & 0 & 0 & 0 & 0 & 0 & 0 & 0 & 0 & 0 \\
\hline Cloudy & 0 & 0 & 0 & 0 & 0 & 0 & 0.1 & 0.2 & 0.3 & 0.4 & 0 \\
\hline Overcas & 0 & 0 & 0 & 0 & 0 & 0 & 0 & 0 & 0.1 & 0.2 & 0.7 \\
\hline
\end{tabular}




\begin{tabular}{|l|l|l|l|l|l|l|l|l|l|l|l|}
\hline $\mathrm{t}$ & & & & & & & & & & & \\
\hline$\ldots$ & $\ldots$ & $\ldots$ & $\ldots$ & $\ldots$ & $\ldots$ & $\ldots$ & $\ldots$ & $\ldots$ & $\ldots$ & $\ldots$ & $\ldots$ \\
\hline
\end{tabular}

\section{Cluster Node Selection.}

Based on fuzzy soft set theory, the method of cluster node selection is proposed as follows:

(1) The fuzzy data set is obtained by normalizing the data of each node. As shown in Table 2. The weights of each node can be calculated.

Table 2. Cluster Node Classification Dataset

\begin{tabular}{|l|l|l|l|l|l|}
\hline node ID & remaining energy & node type & node degree & charge coefficient & $\begin{array}{l}. . \\
.\end{array}$ \\
\hline 1 & 30 & $1 /$ Solar & 0.2 & 0.8 & $\begin{array}{l}. . \\
.\end{array}$ \\
\hline 2 & 5 & $0 /$ Battery & 0.07 & 0 & $\begin{array}{l}. . \\
.\end{array}$ \\
\hline$\ldots$ & & & & & \\
\hline
\end{tabular}

(2) According to the weight ranking, the optimal cluster node is selected and marked.

(3) Each cluster node and its neighbor nodes are divided into a group, and the nature of the nodes in the network is divided. The set of the cluster node represents $\delta_{k}=\left\{x_{k 1}, x_{k 2}, \mathrm{~K}, x_{k n}\right\}$, which is $\mathrm{k}$-degree about neighbor nodes.Parameter $\mathrm{n}$ represents the number of elements in the collection.

(4) According to the position and quantity of the existing cluster nodes in the network, the cluster nodes are re-divided and re-selected, as shown in Figure 1. If there are three or more nodes that are neighbors to each other and that do not belong to any of the existing groups, then they form a new group.

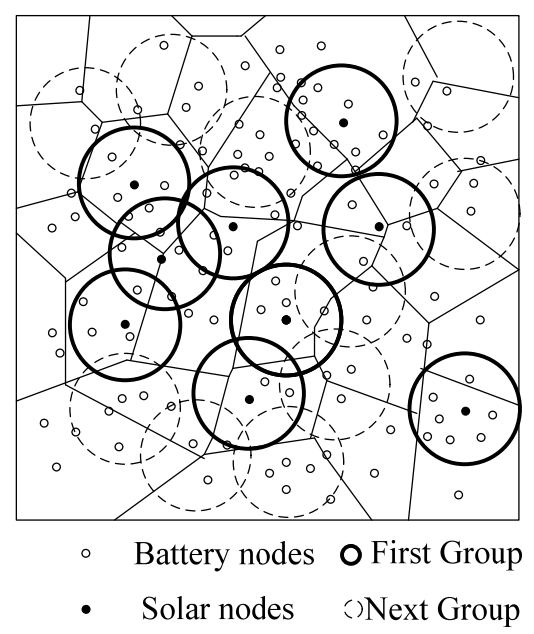

Fig. 1 Group diagram

(5) If all nodes are divided into a cluster or more clusters, the algorithm ends. Otherwise other cluster node is selected, repeat (2).

\section{Analysis}

Experimental Scheme. In this paper, we use Matlab software to build a $100 \mathrm{~m}$ x $100 \mathrm{~m}$ simulation scene. In this area, sink nodes are deployed in the center and 20 solar powered nodes and 80 battery powered nodes are deployed randomly. Other simulation parameters are shown table 3.

Table 3. Simulation Parameters

\begin{tabular}{|l|l|}
\hline Parameters & Value \\
\hline The initial engery of solar and battery power node & $2 \mathrm{~J}$ \\
\hline Data packet size & $1000 \mathrm{bit}$ \\
\hline Node transmission range & $20 \mathrm{~m}$ \\
\hline Transmit power consumption & $50 \mathrm{~nJ}$ \\
\hline Receiving power consumption & $10 \mathrm{~nJ}$ \\
\hline Idle power consumption & $0.013 \mathrm{pJ}$ \\
\hline
\end{tabular}

In this section, we compared the network throughput and network residual energy of EARA and Leach algorithm. 


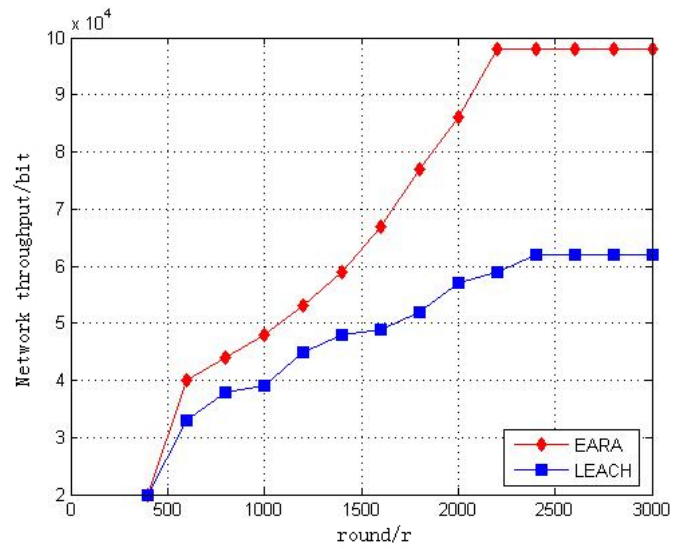

Fig.2 Network throughput

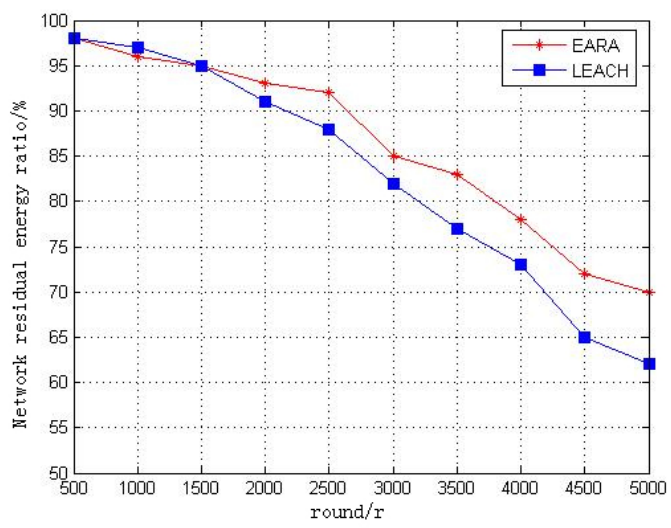

Fig3. Network residual energy

As it can be seen in Fig. 2, the network throughput of EARA is higher than Leach. As the network runs longer, the network throughput converges to a stable value.

Fig. 3 shows the comparison of the WSN remaining energy ratio. In the initial stage, they are almost the same. With the long time of network running, the network remaining energy is decreasing, and the energy difference between the two algorithms becomes larger.

According to the above comparison both network throughput and network residual energy ratio, this paper algorithm than the Leach algorithm has certain advantages.

\section{Conclusion}

FWSN employs the solar power and battery power nodes to prolong network life. According to the forecast of the energy change factor and other indicators, a clustering node selection mechanism is proposed. Experimental results show that this method can be greatly maintained the network thought and prolong the network life. In the future, the accuracy of the prediction about solar energy should be studied due to the farmland environmental monitoring requirements.

\section{Acknowledgements}

This work was financially supported by Natural Science Foundation of China $(61271257,61471067$, $61571051)$.

\section{References}

[1] O. Younis, S. Fahmy. HEED: A Hybrid, Energy-efficient, Distributed Clustering Approach for Ad Hoc Sensor Networks. IEEE Transactions on Mobile Computing, 2004, 3(4): 366-379.

[2] S. Lindsey, C. S. Raghavendra. PEGASIS: Power Efficient Gathering in Sensor Information Systems. In Proceedings of IEEE Aerosapce Conference, 2002:1125-1130.

[3] Roy S. Energy Aware Cluster Based Routing Scheme for Wireless Sensor Network[J]. Foundations of Computing and Decision Sciences, 2015,40(3): 203-222

[4] López J.A, Sotoa F, Suardíaz J. Wireless Sensor Networks for precision horticulture in Southern Spain[J], Computers and Electronics in Agriculture,2006,68( 1),:25-35.

[5] J. Kleissl, "Current Status of the Art in Solar Forecasting," California Renewable Energy Forecasting, Resource Data and Mapping Final Report, Appendix A, 2010.

[6] S. Harrouni and A. Guessoum. Using fractal dimension to quantify long-range persistence in global solar radiation. Chaos, Solitons and Fractal, 41:1520-1530, 2009. 